\title{
Comparison of Three Screening Questionnaires for Chronic Obstructive Pulmonary Disease in the Primary Care
}

\author{
Dionisios Spyratos $^{\text {a }}$ Anna-Bettina Haidich ${ }^{a}$ Diamantis Chloros ${ }^{\mathrm{a}}$ \\ Dionisia Michalopoulou $^{b}$ Lazaros Sichletidis $^{a}$ \\ a Pulmonary Department, Aristotle University of Thessaloniki, "G. Papanikolaou” Hospital, Exohi, and \\ bPrimary Care Center, Municipality of Thessaloniki. Thessaloniki, Greece
}

\section{Keywords}

Chronic obstructive pulmonary disease $\cdot$ Primary care .

Screening questionnaires · Underdiagnosis

\begin{abstract}
Background: Even though the diagnosis of chronic obstructive pulmonary disease (COPD) is easy and based mainly on spirometry and symptoms, the prevalence of underdiagnosis is extremely high. The use of simple screening tools (e.g., questionnaires, hand-held spirometers) has been proved to be a simple method for case finding of COPD. Nevertheless the most appropriate target group of the general population has not been specified yet. Objectives: The aim of the present study was to compare 3 screening questionnaires among smokers aged $>40$ years in the primary care setting. Methods: We excluded all subjects with a previous medical diagnosis of bronchial asthma or chronic pulmonary disease other than COPD. All participants were in a stable clinical condition, filled in the International Primary Care Airways Group (IPAG) questionnaire, the COPD Population Screener (COPDPS) questionnaire, and the Lung Function Questionnaire (LFQ) and underwent spirometry. Medical diagnosis of COPD was established by an experienced pulmonologist. Results: We studied 3,234 subjects during a 3.5-year period. COPD
\end{abstract}

(C) 2017 S. Karger AG, Basel prevalence was $10.9 \%$ (52.1\% underdiagnosis). All 3 questionnaires showed extremely high negative predictive values (94-96\%), so in this case the diagnosis of COPD could be safely excluded. The area under the curve was similar across the 3 questionnaires $\left(A \cup C_{\text {ROC }}\right.$ : 0.794-0.809). The COPD-PS questionnaire demonstrated the highest positive predictive value (41\%) compared to the other 2 . On the other hand, the IPAG questionnaire and LFQ demonstrated higher sensitivities than COPD-PS resulting in lower percentages of missed cases. Conclusions: Three validated screening questionnaires for COPD demonstrated different diagnostic characteristics.

(c) 2017 S. Karger AG, Base

\section{Introduction}

Chronic obstructive pulmonary disease (COPD) is a common disease and one of the main causes of morbidity, mortality and health-related financial burden worldwide [1-3]. It is estimated that it will be the fourth cause of death in 2030 globally [4]. Even though the diagnosis of COPD is easy and based mainly on spirometry and clinical status [1], the prevalence of underdiagnosis is extremely high $->50 \%$ of cases for almost all countries - 
with geographic variation and it is reversely related with the disease severity [5-7]. This phenomenon could be attributed to underestimation of symptoms by the majority of patients and the underuse of spirometry in the primary care [8]. In most countries, general practitioners (GPs) and not pulmonologist are the physicians who initially come in contact, diagnose and treat patients with respiratory symptoms [9]. Even though short-term educational courses of GPs on spirometry could lead to improvement in their diagnostic accuracy for asthma and COPD similar to pulmonologists, adoption of the procedure in everyday clinical routine is not feasible [10].

Increasing age and the presence of respiratory symptoms such as chronic cough and dyspnea during exercise could increase the possibility of COPD diagnosis $[11,12]$. The use of simple screening tools (e.g., questionnaires, hand-held spirometers) that have been validated in the primary care setting has been proved to be an effective method for case finding of COPD [13-18]. A common characteristic of these tools is their high negative predictive value (NPV) - in most studies it exceeds $90 \%$ - while the positive predictive value (PPV) is about $25-35 \%$. The aim of the present study was to compare 3 of the most recently developed questionnaires among a large sample of smokers (exand current), aged $>40$ years in the primary care.

\section{Methods}

Subjects from the general population were invited to participate in the present study by advertisement posters that had been distributed across a network of primary care practices in the city of Thessaloniki, Greece, and in the counties of Pella, Kilkis and Halkidiki covering a radius area of approximately $100 \mathrm{~km}$ around Thessaloniki. The study was performed during a 3.5-year period (January 2012 to June 2015). The Medical Ethics Committee of "G. Papanikolaou” Hospital, Exohi, Thessaloniki, Greece, approved the study protocol.

Participants eligible for this cross-sectional study were subjects aged $>40$ years, current and former smokers ( $\geq 10$ pack-years). Only the inclusion criteria were printed on the posters (age and smoking status) and there was no reference to respiratory symptoms or regular use of inhaled medications.

Exclusion criteria were a previous medical diagnosis of bronchial asthma or chronic pulmonary disease other than COPD (e.g., bronchiectasis, lung cancer, tuberculosis, and interstitial lung disease). Patients with a previous medical diagnosis of COPD who suffered from a recent ( $\leq 8$ weeks) exacerbation leading to hospitalization or prescription of antibiotics and/or corticosteroids were also excluded.

We used 3 different screening COPD questionnaires: (a) the International Primary Care Airways Group (IPAG) questionnaire $[13,14]$ that has already been validated in the Greek population [16], (b) the COPD Population Screener (COPD-PS) question- naire [15], and (c) the Lung Function Questionnaire (LFQ) [17, 18]. We translated the last 2 questionnaires into Greek while the IPAG questionnaire is already in use in the primary care in Greece. We tested the reliability of the COPD-PS questionnaire and LFQ by calculating the reproducibility rate based on the first 120 participants as these 2 questionnaires had not been used in the Greek population in the past (participants filled in the questionnaires on their own twice, 15 days apart). Cutoff points for a positive response were: $\geq 17$ for the IPAG questionnaire, $\geq 5$ for the COPDPS questionnaire and $\leq 18$ for the LFQ based on previous studies.

All subjects filled in the questionnaires unassisted at the primary care clinics (they asked GPs only in case of uncertainties) and they also signed the informed consent form. We recorded all subjects with a previous medical diagnosis of COPD according to their health care booklet. Spirometry was always performed after completion of the questionnaires and informed consent form.

On the same day, all participants underwent spirometry by a group of experienced board-certified pulmonologists (MIR SpiroLab II, Roma, Italy, CE0476, before and 15-30 min after inhalation of $400 \mu \mathrm{g}$ salbutamol via a pressurized metered-dose inhaler with spacer for those with an abnormal prebronchodilation spirometry). Spirometers had been calibrated on the same morning before the visit to the primary care clinic. All measurements were performed according to the criteria of the American Thoracic Society/ European Respiratory Society task force [19]. We used the predicted values for $\mathrm{FVC}, \mathrm{FEV}_{1}$ and $\mathrm{FEF}_{25-75}$ according to the Global Lung Function 2012 equations [20]. All spirometric tests were interpreted by an experienced pulmonologist (criteria for acceptability and reproducibility as well as final diagnostic evaluation of the spirometry) who was blinded to the scores of the 3 COPD screening questionnaires.

Subjects with an $\mathrm{FEV}_{1} / \mathrm{FVC}<0.7$ following bronchodilation were defined as COPD patients according to COLD guidelines [1]. The final medical diagnosis of COPD was based on spirometric criterion, clinical status (medical history, symptoms and physical examination), and exclusion of other diseases [1]. A recent chest $\mathrm{X}$-ray was evaluated for COPD patients, whenever one was available, in order to exclude other undiagnosed diseases. We also collected data for the subgroup of COPD patients about: (a) the modified Medical Research Council (mMRC) Dyspnea Scale and the COPD Assessment Test (CAT) questionnaire score, (b) exacerbations during the last year, and (c) comorbidities that were recorded in the health care booklet. We categorized them to groups A, B, $\mathrm{C}$, and $\mathrm{D}$ based on $\mathrm{FEV}_{1} \%$ predicted, MRC Dyspnea Scale or CAT score, and exacerbation history during the last year.

We retrieved all prescribed inhaled drugs - via the national electronic platform - during the last 12 months for all participants. Overdiagnosis was defined as lung function within predicted values or restriction (post $\mathrm{FEV}_{1} / \mathrm{FVC}>0.7, \mathrm{FCV}$ and $\mathrm{FEV}_{1}<80 \%$ of predicted values), previous medical diagnosis of COPD recorded in the health care booklet, and prescription of inhaled drugs for at least 12 consecutive months during the last 5 years.

\section{Statistical Analysis}

Normally distributed quantitative data were prescribed with mean and standard deviation (SD) and were analyzed within the dependent $t$ test. When data were not normally distributed, they were described with a median and interquartile range (IQR) and analyzed with the Mann-Whitney U test (in the case of comparison between 2 groups) or the Kruskal-Wallis test (in the case of com- 
Table 1. Comparison of demographic, spirometric and screening questionnaire scores between subjects with and without COPD

\begin{tabular}{lccc}
\hline & $\begin{array}{l}\text { Non-COPD subjects } \\
(n=2,883)\end{array}$ & $\begin{array}{l}\text { COPD patients } \\
(n=351)\end{array}$ & $p$ value \\
\hline Gender, $n$ (\%) & & & $<0.001^{\mathrm{a}}$ \\
$\quad$ Males & $1,812(62.9)$ & $290(82.6)$ & \\
$\quad$ Females & $1,071(37.1)$ & $61(17.4)$ & $<0.001^{\mathrm{b}}$ \\
Mean age \pm SD, years & $59 \pm 13.2$ & $65.7 \pm 11.7$ & $<0.001^{\mathrm{b}}$ \\
BMI SD & $29.5 \pm 5.4$ & $28.4 \pm 5.0$ & $0.172^{\mathrm{a}}$ \\
$\quad$ & & \\
$\quad$ Current/former & $1,540(53.4)$ & $201(57.3)$ & $<0.001^{\mathrm{c}}$ \\
Median pack-years (IQR) & $1,343(46.6)$ & $150(42.7)$ & $<0.001^{\mathrm{b}}$ \\
Mean FVC, L (SD) & $24(30)$ & $50(55)$ & $<0.001^{\mathrm{b}}$ \\
Mean FVC \pm SD, \%pred. & $3.36 \pm 1.03$ & $3.02 \pm 1.0$ & $<0.001^{\mathrm{b}}$ \\
Mean FEV \pm SD, L & $95 \pm 17.6$ & $1.9 \pm 0.7$ & $<0.001^{\mathrm{b}}$ \\
FEV \pm SD, \%pred. & $2.8 \pm 0.8$ & $68.5 \pm 18.8$ & $<0.001^{\mathrm{c}}$ \\
Median FEV $/$ FVC, \% (IQR) & $97.4 \pm 17.0$ & $64.9(7.8)$ & $<001^{\mathrm{c}}$ \\
Median MMEF, \%pred. (IQR) & $82.9(10)$ & $38.7(16.1)$ & $<0.001^{\mathrm{b}}$ \\
Mean score IPAG \pm SD [13, 14] & $90.1(41.7)$ & $21.1 \pm 6.7$ & $<0.001^{\mathrm{b}}$ \\
Mean score COPD-PS \pm SD [15] & $13.5 \pm 5.8$ & $5.4 \pm 2.1$ & $<0.001^{\mathrm{b}}$ \\
Mean score LFQ \pm SD [17, 18] & $3.3 \pm 1.6$ & $15 \pm 4.1$ &
\end{tabular}

SD, standard deviation; IQR, interquartile range. ${ }^{a}$ Pearson $\chi^{2} .{ }^{b}$ Independent $t$ test. ${ }^{c}$ Mann-Whitney test.

parison between 3 groups). The Bonferroni correction was applied for multiple comparisons. The Pearson $\chi^{2}$ test was used for qualitative data. The intraclass correlation coefficient was calculated to evaluate the reproducibility rate between the same questionnaires. The $95 \%$ confidence intervals were calculated for the diagnostic proportions results. The Youden index was used to find the optimal cutoff point for sensitivity and specificity. The Delong test was used for comparing 2 areas under the curve (AUCs) except for comparison of curves with different direction, where the bootstrap method was used. The confidence level was set as $\alpha=0.05$. A subgroup analysis was performed excluding those with a previous medical diagnostic of COPD and receiving inhaled drugs at the time of the study. The statistical analysis was conducted using $\mathrm{R}$ and SPSS statistical packages.

\section{Results}

The study sample consisted of 3,319 subjects totally. Eighty-five participants were excluded because their spirometric maneuvers did not fulfill the American Thoracic Society/European Respiratory Society criteria. The eligible sample for the final analysis consisted of 3,234 subjects (65\% males, 54\% current and $46 \%$ former smokers).

A subgroup of 351 patients (10.9\%) presented with clinical symptoms as well as postbronchodilation spirometric values compatible with a diagnosis of COPD. De- mographics, spirometric values and scores of the 3 screening questionnaires of the subjects with and without COPD are shown in Table 1. Patients with COPD achieved worse scores across all questionnaires as it was anticipated. One hundred and eighty-three patients had no previous medical diagnosis of COPD (underdiagnosed, 52.1\%), while 168 (47.9\%) had already been diagnosed and were receiving inhaled drugs at the time of the study. Overdiagnosis was the case for 307 subjects (9.5\%).

We also estimated severity according to the combined COPD assessment system as proposed by GOLD (spirometry, exacerbation rate, and symptoms). The patients were divided into group A (240 patients), group B (58 patients), group C (20 patients), and group D (33 patients) based on the mMRC Dyspnea Scale. On the other hand, using the CAT for evaluating symptoms, we estimated the following classification: group A (160 patients), group B (138 patients), group C (14 patients), and group D (39 patients). Comorbidities of COPD patients were: arterial hypertension $(n=133,37.9 \%)$, coronary artery disease $(n=73,20.8 \%)$, hyperlipidemia $(n=60,17.1 \%)$, diabetes $(n=48,13.7 \%)$, depression $(n=23,6.6 \%)$, and cerebrovascular accident $(n=13,3.7 \%)$.

The reproducibility rate of the COPD-PS questionnaire and LFQ was calculated to be $89 \%$ (95\% CI: 83-95) 
Table 2. Characteristics of the 3 case-finding COPD questionnaires for the whole study sample

\begin{tabular}{|c|c|c|c|c|}
\hline Sensitivity & $0.74(0.69-0.79)$ & $0.56(0.51-0.61)$ & $0.79(0.75-0.84)$ & \\
\hline Specificity & $0.72(0.70-0.74)$ & $0.90(0.89-0.91)$ & $0.68(0.66-0.69)$ & \\
\hline PPV & $0.24(0.22-0.27)$ & $0.41(0.37-0.45)$ & $0.23(0.21-0.26)$ & \\
\hline NPV & $0.96(0.95-0.97)$ & $0.94(0.93-0.95)$ & $0.96(0.96-0.97)$ & \\
\hline Diagnostic accuracy & $0.72(0.71-0.74)$ & $0.86(0.85-0.88)$ & $0.69(0.67-0.71)$ & \\
\hline QA-QC (bootstrap method, $n=10,000$ ) & & & & $p=0.4085$ \\
\hline QB-QC (bootstrap method, $n=10,000$ ) & & & & $p=0.0613$ \\
\hline \multicolumn{5}{|l|}{ Best cutoff point } \\
\hline Based on Youden method & 19.5 & 4.5 & 17.5 & \\
\hline Based on Closest Top Left method & 16.5 & 4.5 & 17.5 & \\
\hline
\end{tabular}

QA, IPAG questionnaire; QB, COPD-PS questionnaire; QC, LFQ.

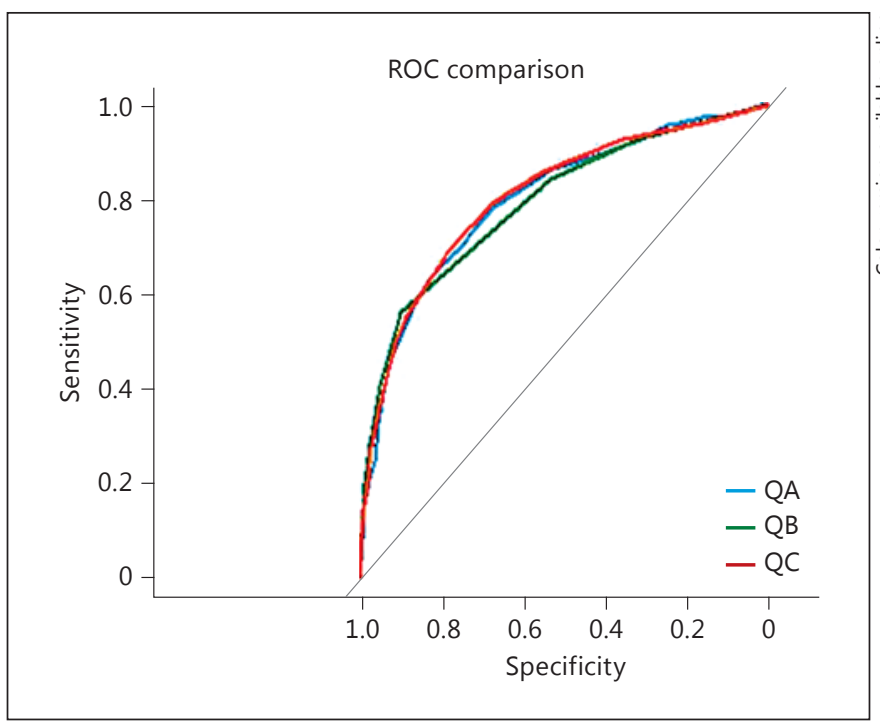

Fig. 1. ROC curves for the 3 questionnaires analyzing the whole study sample. QA, International Primary Care Airways Group questionnaire; QB, Chronic Obstructive Pulmonary Disease Population Screener questionnaire; QC, Lung Function Questionnaire.

and 91\% (95\% CI: 88-97), respectively, based on a subsample of the first 120 subjects who filled in the questionnaires twice ( 15 days apart).

As it is shown in Table 2, the possibility of a patient to suffer from the disease was higher if he/she had a positive COPD-PS questionnaire (PPV $=41 \%$ compared with 23 and $24 \%$ for the other 2 questionnaires). On the other hand, it is important to mention that all 3 questionnaires

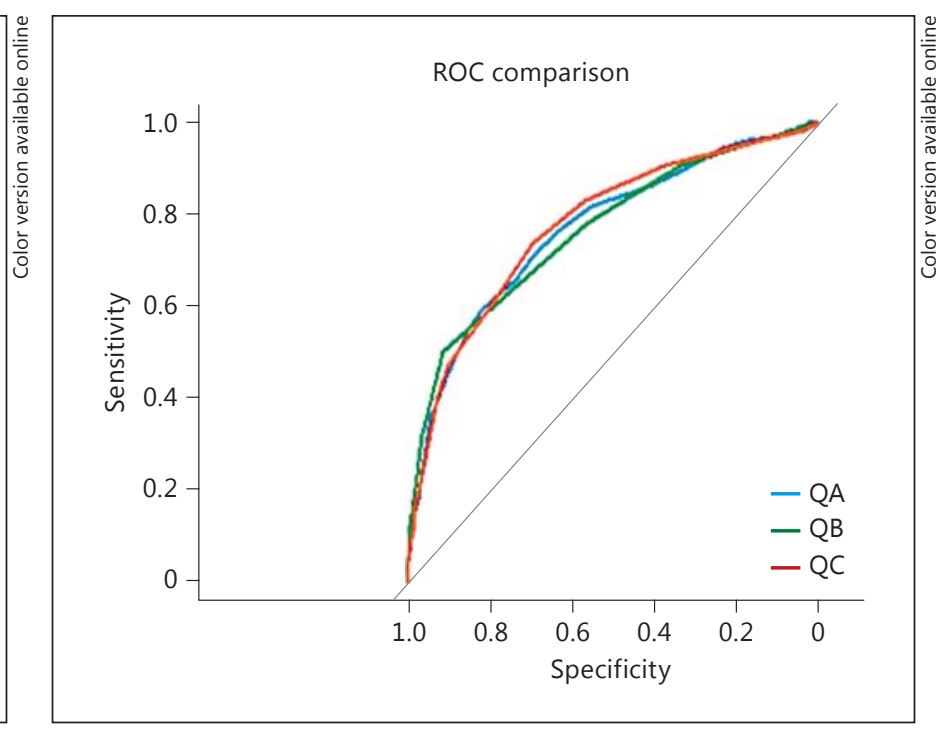

Fig. 2. ROC curves for the 3 questionnaires after excluding subjects with a previous medical diagnosis of COPD. QA, International Primary Care Airways Group questionnaire; QB, Chronic Obstructive Pulmonary Disease Population Screener questionnaire; QC, Lung Function Questionnaire.

had extremely high NPVs (94-96\%) so in this case the diagnosis of COPD could be quite safely excluded. The AUC was similar across the 3 questionnaires as it is shown by the ROC curves (Fig. 1). We also calculated the same diagnostic characteristics of the 3 questionnaires after excluding those with a previous medical diagnosis of COPD and those receiving inhaled drugs (correctly diagnosed and those with an overdiagnosis) at the time of the study
86

Respiration 2017;93:83-89 DOI: $10.1159 / 000453586$
Spyratos/Haidich/Chloros/ Michalopoulou/Sichletidis 
Table 3. Characteristics of the 3 case-finding COPD questionnaires after excluding those with a previous medical diagnosis of COPD and those receiving inhaled drugs

\begin{tabular}{lllll}
\hline & IPAG $(\geq 17)$ & COPD-PS $(\geq 5)$ & LFQ $(\leq 18)$ & Comparison of AUC \\
\hline Sensitivity & $0.66(0.59-0.73)$ & $0.50(0.43-0.58)$ & $0.74(0.67-0.80)$ \\
Specificity & $0.74(0.72-0.75$ & $0.91(0.90-0.93)$ & $0.69(0.68-0.71)$ & $0.15(0.12-0.17)$ \\
PPV & $0.15(0.13-0.18)$ & $0.29(0.24-0.35)$ & $0.97(0.97-0.98)$ \\
NPV & $0.97(0.96-0.98)$ & $0.96(0.95-0.97)$ & $0.70(0.68-0.71)$ & $z=0.195, p=0.845$ \\
Diagnostic accuracy & $0.73(0.71-0.75)$ & $0.89(0.88-0.90)$ & $p=0.5163$ \\
\hline AUC & $0.769(0.731-0.807)$ & $0.766(0.727-0.805)$ & $0.777(0.741-0.814)$ & $p=0.3264$ \\
$\quad$ & & & 18.5 \\
$\quad$ QA-QB (Delong test) & & & 18.5 & \\
$\quad$ QB-QC (bootstrap method, $n=10,000)$ & & & \\
\hline Best cutoff point & & 4.5 & 3.5 & \\
$\quad$ Based on Youden method & & & \\
Based on Closest Top Left method & 18.5 & & & \\
\hline
\end{tabular}

QA, IPAG questionnaire; QB, COPD-PS questionnaire; QC, LFQ.

Table 4. Questionnaire scores for subgroups of diagnosed and underdiagnosed COPD patients as well as overdiagnosed subjects

$\begin{array}{llll}\begin{array}{l}\text { Patients with previous } \\ \text { diagnosis of COPD }(n=168)\end{array} & \begin{array}{l}\text { Underdiagnosed patients } \\ \text { with COPD }(n=183)\end{array} & \begin{array}{l}\text { Overdiagnosed } \\ \text { subjects }(n=307)\end{array} & p \text { value* } \\ & & & \\ 22.5 \pm 6.2 ; 23(9) & 19.7 \pm 6.9 ; 20(9) & 16.3 \pm 6.1 ; 15(8) & <0.001 \\ 139 / 168(82.7 \%) & 121 / 183(66.1 \%) & 128 / 307(41.7 \%) & \\ & & & <0.001 \\ 5.9 \pm 2.3 ; 5.5(4) & 4.9 \pm 1.9 ; 5(2) & 4 \pm 1.7 ; 4(1) & <0.001 \\ 105 / 168(62.5 \%) & 92 / 183(50.3 \%) & 64 / 307(20.8 \%) & \\ 13.9 \pm 4.2 ; 14(6) & 16.1 \pm 3.6 ; 17(5) & 18.1 \pm 3.2 ; 19(3) & 139 / 307(45.3 \%) \\ 144 / 168(85.7 \%) & 135 / 183(73.8 \%) & & \end{array}$

Score IPAG questionnaire; median (IQR)

Patients with a positive QA

Score COPD-PS questionnaire; median (IQR)

Patients with a positive QB

Score LFQ; median (IQR)

Patients with a positive QC

QA, IPAG questionnaire; QB, COPD-PS questionnaire; QC, LFQ. All data are presented as mean values \pm SD if not otherwise noted. * Kruskal-Wallis test, multiple pairwise comparisons (using the Mann-Whitney test with Bonferroni correction) found significant differences $(p<0.001)$ for all comparisons.

$(n=2,676$, COPD prevalence $=186 / 2,676=6.8 \%) . \mathrm{We}$ performed this subgroup analysis as these subjects did not have any previous medical diagnosis of respiratory disease and may seek support in case of worrisome symptoms in the future. The results were similar as the 3 questionnaires had a comparable AUC and NPV, while the COPD-PS questionnaire had a better PPV (Table 3; Fig. 2). We performed another subgroup analysis based on patients with COPD stages B, C, and D as they need regular treatment with inhaled drugs according to the GOLD guidelines. We also found that the COPD-PS questionnaire had a better PPV (0.33) compared to the other 2 ( 0.19 for the IPAG and 0.18 for the LFQ).

Comparing Screening Questionnaires for COPD
Trying to determine the best cutoff point (the Youden index method calculating the maximal combination of sensitivity and specificity), we found that the previously proposed scores for a positive test $[14,15,17]$ were exactly the same as in the present study for the COPD-PS questionnaire and the LFQ. We also calculated that the best cutoff point for the IPAG questionnaire should be raised to $\geq 19$ based on the Youden method (Table 3 ).

It is interesting to mention that the subgroup with an overdiagnosis of COPD had the lowest scores across all questionnaires while those with a previous correct diagnosis achieved the highest scores (Table 4). On the other hand, patients with an underdiagnosis of COPD were fre- 
quently symptomatic (50-74\% had positive questionnaires) even though they had not sought medical advice until the time of the study.

\section{Discussion}

Simple questionnaires that are easy for everyone to understand and complete are useful tools in order to identify patients at risk for COPD. National and international websites related with COPD could use these questionnaires as a first-level screening method of the general population and advice subjects with a positive test to visit their physician and perform spirometry.

In the present study, we compared the ability of 3 different screening questionnaires to discriminate between subjects with and without COPD. The main findings are: (a) overall the diagnostic characteristics were similar among the 3 questionnaires (diagnostic accuracy and AUC), (b) the NPV for all questionnaires was extremely high making spirometry unnecessary in that case, (c) the IPAG questionnaire and the LFQ demonstrated higher sensitivities than the COPD-PS questionnaire resulting in lower percentages of missed cases, and (d) the cutoff point for the IPAG questionnaire should be raised to 19 points in order to improve its diagnostic accuracy. To our current knowledge, this is the largest population-based study on case finding of COPD patients using different screening questionnaires among smokers (current and former) in the primary care.

Price et al. [13] developed the IPAG questionnaire based on 818 subjects, $45 \%$ current smokers, with no prior history of respiratory disease and COPD prevalence of $18.6 \%$. Using the cutoff point of 17 [14], the questionnaire had a sensitivity of $58.7 \%$, a specificity of $77 \%$, an NPV of $89 \%$, a PPV of $37 \%$ and an $\mathrm{ROC}_{\mathrm{AUC}}$ of 0.8158 . In our study, the $\mathrm{ROC}_{\mathrm{AUC}}$ was quite similar (0.802 and 0.769) based on the whole study sample (COPD prevalence: $10.9 \%$ ) and after excluding those with prior COPD diagnosis (COPD prevalence: $6.8 \%$ ). Using the above questionnaire as a telephone interview is also possible and the percentage of new COPD cases was higher among those who achieved the high-risk score [21]. On the other hand, we should mention that an external validation study of the IPAG questionnaire [22] showed that its usefulness was too low $\left(\mathrm{ROC}_{\mathrm{AUC}} 0.65\right)$ among 676 current smokers with a high prevalence of COPD (41.1\%).

Martinez et al. [15] studied 295 subjects - 38\% of whom had persistent airflow obstruction - and developed the COPD-PS questionnaire which achieved an $\mathrm{ROC}_{\mathrm{AUC}}$ of 0.73 ( 0.794 in the present study) for the cutoff point of 5. Hanania et al. [18] showed that the main advantage of the LFQ was its high sensitivity (82.6\%), although it was not too specific. In everyday clinical practice, this means that it could identify more patients with COPD but at the same time more subjects should undergo spirometry because of a positive test.

The low COPD prevalence in the present study could give an explanation for the differences in PPV. We calculated that the LFQ had a PPV of 0.23 , which was very close to the original study $(0.26)$ as the prevalence of COPD was quite similar (10.9 vs. $18.6 \%)$. We should mention that the COPD prevalence of our study was comparable with that of the BOLD study [2].

Screening questionnaires is a validated way for finding new cases of COPD; however, offering spirometry to subjects with tobacco/occupational exposure and at least one respiratory symptom (e.g., dyspnea, cough, wheeze, and phlegm) seems to be a very effective screening strategy. One study performed by the Danish GPs who followed the above criteria found that more than one-third of participants had airway obstruction [23]. The same screening program seems to be feasible and effective if implemented out of general practices (e.g., municipalities) [24]. Additional screening tools such as differential diagnosis questionnaires for those with prior evidence of respiratory medical history have been developed for use in the primary care [25].

Screening programs and ongoing education of GPs to use simple tools among high-risk populations in primary care is the only feasible way to reduce the percentage of underdiagnosed COPD. At the same time, finding mild and moderate COPD cases may increase the long-term effectiveness of inhaled drugs in altering the natural history of the disease.

\section{Financial Disclosure and Conflicts of Interest}

All authors state that they do not have any financial or other relationships for the present study that might lead to a conflict of interest.
Spyratos/Haidich/Chloros/ Michalopoulou/Sichletidis 


\section{References}

1 Global Initiative for the Chronic Obstructive Lung Disease. Global Strategy for the Diagnosis, Management, and Prevention of Chronic Obstructive Pulmonary Disease (updated 2015). www.goldcopd.org.

2 Buist AS, McBurnie MA, Vollmer WM, Gillespie S, Burney P, Mannino DM, et al: International variation in the prevalence of COPD (the BOLD Study): a population-based prevalence study. Lancet 2007;370:741-750.

3 Foster TS, Miller JD, Marton JP, et al: Assessment of the economic burden of COPD in the US: a review and synthesis of the literature. COPD 2006;3:211-218.

4 Mathers CD, Loncar D: Projections of global mortality and burden of disease from 2002 to 2030. PLoS Med 2006;3:e442.

5 Peña VS, Miravitlles M, Gabriel R, JiménezRuiz CA, Villasante C, Masa JF, Viejo JL, Fernández-Fau L: Geographic variations in prevalence and underdiagnosis of COPD: results of the IBERPOC multicentre epidemiological study. Chest 2000;118:981-989.

6 Lamprecht B, Soriano JB, Studnicka M, Kaiser B, Vanfleteren LE, Gnatiuc L, et al: Determinants of underdiagnosis of COPD in National and International Surveys. Chest 2015;148: 971-985.

7 Lindberg A, Bjerg A, Rönmark E, Larsson LG, Lundbäck B: Prevalence and underdiagnosis of COPD by disease severity and the attributable fraction of smoking Report from the Obstructive Lung Disease in Northern Sweden Studies. Respir Med 2006;100:264-272.

8 Arne M, Lisspers K, Ställberg B, Boman G, Hedenström $\mathrm{H}$, Janson C, Emtner M: How often is diagnosis of COPD confirmed with spirometry? Respir Med 2010;104:550-556.

9 van Weel C: Underdiagnosis of asthma and COPD: is the general practitioner to blame? Monaldi Arch Chest Dis 2002;57:65-68.

10 Lusuardi M, De Benedetto F, Paggiaro P, Sanguinetti CM, Brazzola G, Ferri P, Donner CF:
A randomized controlled trial on office spirometry in asthma and COPD in standard general practice: data from spirometry in asthma and COPD: a comparative evaluation Italian study. Chest 2006;129:844-852.

11 Van Schayck CP, Loozen JM, Wagena E, Akkermans RP, Wesseling GJ: Detecting patients at a high risk of developing chronic obstructive pulmonary disease in general practice: cross sectional case finding study. BMJ 2002;324:1370

12 Freeman D, Nordyke RJ, Isonaka S, Nonikov DV, Maroni JM, Price D, Halbert RJ: Questions for COPD diagnostic screening in a primary care setting. Respir Med 2005;99:13111318.

13 Price DB, Tinkelman DG, Halbert RJ, Nordyke RJ, Isonaka $S$, Nonikov D, Juniper EF, Freeman D, Hausen T, Levy ML, Ostrem A, van der Molen T, van Schayck CP: Symptombased questionnaire for identifying COPD in smokers. Respiration 2006;73:285-295.

14 Price DB, Tinkelman DG, Nordyke RJ, Isonaka S, Halbert RJ; COPD Questionnaire Study Group: Scoring system and clinical application of COPD diagnostic questionnaires. Chest 2006;129:1531-1539.

15 Martinez FJ, Raczek AE, Seifer FD, Conoscenti CS, Curtice TG, D'Eletto T, et al: Development and initial validation of a self-scored COPD Population Screener Questionnaire (COPD-PS). COPD 2008;5:85-95.

16 Sichletidis L, Spyratos D, Papaioannou M, Chloros D, Tsiotsios A, Tsagaraki V, Haidich $A B$ : A combination of the IPAG questionnaire and PiKo- $6^{\circledR}$ flow meter is a valuable screening tool for COPD in the primary care setting. Prim Care Respir J 2011;20:184-189.

17 Yawn BP, Mapel DW, Mannino DM, Martinez FJ, Donohue JF, Hanania NA, Kosinski M, Rendas-Baum R, Mintz M, Samuels S, Dalal AA; Lung Function Questionnaire Working Group: Development of the Lung Func- tion Questionnaire (LFQ) to identify airflow obstruction. Int J Chron Obstruct Pulmon Dis 2010;5:1-10.

18 Hanania NA, Mannino DM, Yawn BP, Mapel DW, Martinez FJ, Donohue JF, Kosinski M, Rendas-Baum R, Mintz M, Samuels S, Jhingran P, Dalal AA: Predicting risk of airflow obstruction in primary care: validation of the lung function questionnaire (LFQ). Respir Med 2010;104:1160-1170.

19 Miller MR, Hankinson J, Brusasco V, et al Standardisation of spirometry. Eur Respir J 2005;26:319-338.

20 Quanjer PH, Stanojevic S, Cole TJ, Baur X, Hall GL, Culver BH, Enright PL, Hankinson JL, Ip MS, Zheng J, Stocks J; ERS Global Lung Function Initiative: Multi-ethnic reference values for spirometry for the 3-95-yr age range: the global lung function 2012 equations. Eur Respir J 2012;40:1324-1343.

21 Dirven JA, Muris JW, van Schayck CP: COPD screening in general practice using a telephone questionnaire. COPD 2010;7:352359

22 Kotz D, Nelemans P, van Schayck CP, Wesseling GJ: External validation of a COPD diagnostic questionnaire. Eur Respir J 2008;31: 298-303.

23 Ulrik CS, Løkke A, Dahl R, Dollerup J, Hansen G, Cording PH, Andersen KK; TOP study group: Early detection of COPD in general practice. Int J Chron Obstruct Pulmon Dis 2011;6:123-127.

24 Riegels-Jakobsen T, Skouboe M, Dollerup J, Andersen CB, Staal LB, Jakobsen RB, Poulsen PB: Municipality screening of citizens with suspicion of chronic obstructive pulmonary disease. Int J Chron Obstruct Pulmon Dis 2012;7:35-41.

25 Tinkelman DG, Price DB, Nordyke RJ, et al: Symptom-based questionnaire for differentiating COPD and asthma. Respiration 2006; 73:296-305. 